\title{
Preface: Selected Extended Papers of CADE 2017
}

\author{
Leonardo de Moura $^{1}$
}

Received: 27 February 2019 / Accepted: 5 February 2020 / Published online: 17 February 2020 (c) Springer Nature B.V. 2020

This special issue of the Journal of Automated Reasoning is dedicated to selected papers presented at the 26th Edition of the International Conference on Automated Deduction, held between August 6 and 11 in Gothenburg, Sweden. CADE is the major forum for the presentation of research in all aspects of automated deduction, including foundations, applications, implementations, and practical experience. The five papers selected for the special issue (out of 31 papers presented at the conference) underwent a new and thorough reviewing and revision process, in accordance with the high standards of JAR.

The paper Efficient Verified (UN)SAT Certificate Checking by Peter Lammich presents an efficient and formally verified checker for satisfiability and unsatisfiability certificates for Boolean formulas in conjunctive normal form.

Marijn Heule, Benjamin Kiesl and Armin Biere present Strong Extension-Free Proof Systems. It introduces proof systems for propositional logic that admit short proofs of hard formulas as well as the succinct expression of most techniques used by modern SAT solvers. The conference version of this paper was awarded the CADE-26 Best paper award.

In the paper Automatically Verifying Temporal Properties of Pointer Programs with Cyclic Proof, Gadi Tellez and James Brotherston investigate the automated verification of temporal properties of heap-aware programs. They propose a deductive reasoning approach based on cyclic proof.

The paper Conflict-Driven Satisfiability for Theory Combination: Transition System and Completeness by Maria Paola Bonacina, Stéphane Graham-Lengrand and Natarajan Shankar, presents a new method for satisfiability modulo a combination of theories, named ConflictDriven Satisfiability (CDSAT).

Andreas Teucke and Christoph Weidenbach present SPASS-AR: A First-Order Theorem Prover based on Approximation-Refinement into the Monadic Shallow Linear Fragment. It introduces FO-AR, an approximation-refinement approach for first-order theorem proving based on counterexample-guided abstraction refinement.

We thank the authors for their contributions and the reviewers for their thorough and extensive feedback.

Publisher's Note Springer Nature remains neutral with regard to jurisdictional claims in published maps and institutional affiliations.

Leonardo de Moura

leonardo@microsoft.com

1 Microsoft Research, One Microsoft Way, Redmond, WA 98052, USA 